\title{
Common fixed-point results for nonlinear contractions in ordered partial metric spaces
}

\author{
Bessem Samet ${ }^{1 *}$, Miloje Rajović ${ }^{2}$, Rade Lazović ${ }^{3}$ and Rade Stojiljković ${ }^{4}$
}

\author{
* Correspondence: bessem. \\ samet@gmail.com \\ 'Université de Tunis, Ecole \\ Supérieure des Sciences et \\ Techniques de Tunis, 5, Avenue \\ Taha Hussein-Tunis, B.P.:56, 1008 \\ Bab Menara, Tunisia \\ Full list of author information is \\ available at the end of the article
}

\begin{abstract}
In this paper, a new class of a pair of generalized nonlinear contractions on partially ordered partial metric spaces is introduced, and some coincidence and common fixed-point theorems for these contractions are proved. Presented theorems are twofold generalizations of very recent fixed-point theorems of Altun and Erduran (Fixed Point Theory Appl 2011(Article ID 508730):10, 2011), Altun et al. (Topol Appl 157(18):2778-2785, 2010), Matthews (Proceedings of the 8th summer conference on general topology and applications, New York Academy of Sciences, New York, pp. 183-197, 1994) and many other known corresponding theorems.

2000 Mathematics Subject Classifications: 54H25; 47H10.
\end{abstract}

Keywords: partial metric, ordered set, common fixed point, coincidence point, partial compatible

\section{Introduction}

It is well known that the Banach contraction principle is a very useful, simple and classical tool in nonlinear analysis. There exist a vast literature concerning its various generalizations and extensions (see [1-45]). In [22], Matthews extended the Banach contraction mapping theorem to the partial metric context for applications in program verification. After that, fixed-point results in partial metric spaces have been studied $[4,8,28,31,34,45]$. The existence of several connections between partial metrics and topological aspects of domain theory has been pointed by many authors (see $[8,9,16,23,31,33,36-38,41,42,46,47])$.

First, we recall some definitions of partial metric spaces and some their properties.

Definition 1.1 A partial metric on a set $X$ is a function $p: X \times X \rightarrow \mathbb{R}^{+}$such that for all $x, y, z \in X$ :

$$
\begin{aligned}
& \text { (p1) } x=y \Leftrightarrow p(x, x)=p(x, y)=p(y, y), \\
& \text { (p2) } p(x, x) \leq p(x, y), \\
& \text { (p3) } p(x, y)=p(y, x), \\
& \text { (p4) } p(x, y) \leq p(x, z)+p(z, y)-p(z, z) .
\end{aligned}
$$

Note that the self-distance of any point need not be zero, hence the idea of generalizing metrics so that a metric on a non-empty set $X$ is precisely a partial metric $p$ on $X$ such that for any $x \in X, p(x, x)=0$.

Similar to the case of metric space, a partial metric space is a pair $(X, p)$ consisting of a non-empty set $X$ and a partial metric $p$ on $X$.

(c) 2011 Samet et al; licensee Springer. This is an Open Access article distributed under the terms of the Creative Commons Attribution License (http://creativecommons.org/licenses/by/2.0), which permits unrestricted use, distribution, and reproduction in any medium, provided the original work is properly cited. 
Example 1.1 Let a function $p: \mathbb{R}^{+} \times \mathbb{R}^{+} \rightarrow \mathbb{R}^{+}$be defined by $p(x, y)=\max \{x, y\}$ for any $x, y \in \mathbb{R}^{+}$. Then, $\left(\mathbb{R}^{+}, p\right)$ is a partial metric space where the self-distance for any point $x \in \mathbb{R}^{+}$is its value itself.

Example 1.2 Consider a function $p: \mathbb{R}^{-} \times \mathbb{R}^{-} \rightarrow \mathbb{R}^{+}$defined by $p(x, y)=-\min (x, y)$ for any $x, y \in \mathbb{R}^{-}$. The pair $\left(\mathbb{R}^{-}, p\right)$ is a partial metric space for which $p$ is called the usual partial metric on $\mathbb{R}^{-}$and where the self-distance for any point $x \in \mathbb{R}^{-}$is its absolute value.

Example 1.3 If $X:=\{[a, b] \mid a, b \in \mathbb{R}, a \leq b\}$, then $p: X \times X \rightarrow \mathbb{R}^{+}$defined by $p([a$, $b],[c, d])=\max \{b, d\}-\min \{a, b\}$ defines a partial metric on $X$.

Each partial metric $p$ on $X$ generates a $T_{0}$ topology $\tau_{p}$ on $X$, which has as a base the family of open $p$-balls $\left\{B_{p}(x, \varepsilon), x \in X, \varepsilon>0\right\}$, where

$$
B_{p}(x, \varepsilon)=\{y \in X \mid p(x, y)<p(x, x)+\varepsilon\} \text { for all } x \in X \text { and } \varepsilon>0 .
$$

If $p$ is a partial metric on $X$, then the function $p^{s}: X \times X \rightarrow \mathbb{R}^{+}$defined by

$$
p^{s}(x, y)=2 p(x, y)-p(x, x)-p(y, y)
$$

is a metric on $X$.

Definition 1.2 Let $(X, p)$ be a partial metric space and $\left\{x_{n}\right\}$ be a sequence in $X$. Then,

(i) $\left\{x_{n}\right\}$ converges to a point $x \in X$ if and only if $p(x, x)=\lim _{n \rightarrow+\infty} p\left(x, x_{n}\right)$,

(ii) $\left\{x_{n}\right\}$ is a Cauchy sequence if there exists (and is finite) $\lim _{n, m \rightarrow+\infty} p\left(x_{n}, x_{m}\right)$.

Definition 1.3 A partial metric space $(X, p)$ is said to be complete if every Cauchy sequence $\left\{x_{n}\right\}$ in $X$ converges, with respect to $\tau_{p}$, to a point $x \in X$, such that $p(x, x)=$ $\lim _{n, m \rightarrow+\infty} p\left(x_{n}, x_{m}\right)$.

Remark 1.1 It is easy to see that every closed subset of a complete partial metric space is complete.

Lemma $1.1([22,28])$ Let $(X, p)$ be a partial metric space. Then

(a) $\left\{x_{n}\right\}$ is a Cauchy sequence in $(X, P)$ if and only if it is a Cauchy sequence in the metric space $\left(X, P^{s}\right)$,

(b) $(X, p)$ is complete if and only if the metric space $\left(X, p^{s}\right)$ is complete. Furthermore, $\lim _{n \rightarrow+\infty} p^{s}\left(x_{n}, x\right)=0$ if and only if

$$
p(x, x)=\lim _{n \rightarrow+\infty} p\left(x_{n}, x\right)=\lim _{n, m \rightarrow+\infty} p\left(x_{n}, x_{m}\right) .
$$

Matthews [22] obtained the following Banach fixed-point theorem on complete partial metric spaces.

Theorem 1.1 (Matthews [22]) Let $f$ be a mapping of a complete partial metric space $(X, p)$ into itself such that there is a constant $c \in[0,1)$ satisfying for all $x, y \in X$ :

$$
p(f x, f y) \leq c p(x, y) .
$$

Then, $f$ has a unique fixed point.

Recently, Altun et al. [4] obtained the following nice result, which generalizes Theorem 1.1 of Matthews.

Theorem 1.2 (Altun et al. [4]) Let $(X, p)$ be a complete partial metric space and let $T: X \rightarrow X$ be a map such that

$$
p(T x, T y) \leq \varphi\left(\max \left\{p(x, y), p(x, T x), p(y, T y), \frac{1}{2}[p(x, T y)+p(y, T x)]\right\}\right)
$$


for all $x, y \in X$, where $\phi:[0,+\infty) \rightarrow[0,+\infty)$ satisfies the following conditions:

(i) $\phi$ is continuous and non-decreasing,

(ii) $\sum_{n \geq 1} \varphi^{n}(t)$ is convergent for each $t>0$.

Then, $T$ has a unique fixed point.

On the other hand, existence of fixed points in partially ordered sets has been considered recently in [32], and some generalizations of the result of [32] are given in [1-3,5-7,11,12,14,15,17,19,24-27,29,30,39,40,43] in partial ordered metric spaces. Also, in [32], some applications to matrix equations are presented, and in [15] and [26], some applications to ordinary differential equations are given. In [29], O’Regan and Petruşel established some fixed-point results for self-generalized contractions in ordered metric spaces. Jachymski [19] established a geometric lemma [19, Lemma 1], giving a list of equivalent conditions for some subsets of the plane. Using this lemma, he proved that some very recent fixed-point theorems for generalized contractions on ordered metric spaces obtained by Harjani and Sadarangani [15] and Amini-Harandi and Emami [5] do follow from an earlier result of O’Regan and Petruşel [29, Theorem 3.6].

Very recently, Altun and Erduran [3] generalized Theorem 1.2 to partially ordered complete partial metric spaces and established the following new fixed-point theorems, involving a function $\phi:[0,+\infty) \rightarrow[0,+\infty)$ satisfying the conditions (i)-(ii) in Theorem 1.2 .

Theorem 1.3 (Altun and Erduran [3]). Let $(X, \preccurlyeq)$ be a partially ordered set and suppose that there is a partial metric $p$ on $X$ such that $(X, p)$ is a complete partial metric space. Suppose $F: X \rightarrow X$ is a continuous and non-decreasing mapping (with respect to ミ) such that

$$
p(F x, F y) \leq \varphi\left(\max \left\{p(x, y), p(x, F x), p(y, F y), \frac{1}{2}[p(x, F y)+p(y, F x)]\right\}\right)
$$

for all $x, y \in X$ with $y \leqslant x$, where $\phi:[0,+\infty) \rightarrow[0,+\infty)$ satisfies conditions (i)-(ii) in Theorem 1.2. If there exists $x_{0} \in X$ such that $x_{0} \leqslant F x_{0}$, then there exists $x \in X$ such that $F x=x$. Moreover, $p(x, x)=0$.

Theorem 1.4 (Altun and Erduran [3]) Let $(X, \preccurlyeq)$ be a partially ordered set and suppose that there is a partial metric $p$ on $X$ such that $(X, p)$ is a complete partial metric space. Suppose $F: X \rightarrow X$ is a non-decreasing mapping such that

$$
p(F x, F y) \leq \varphi\left(\max \left\{p(x, y), p(x, F x), p(y, F y), \frac{1}{2}[p(x, F y)+p(y, F x)]\right\}\right)
$$

for all $x, y \in X$ with $y<x(y \leqslant x$ and $y \neq x)$, where $\phi:[0,+\infty) \rightarrow[0,+\infty)$ satisfies conditions (i)-(ii) in Theorem 1.2. Suppose also that the condition

$$
\left\{\begin{array}{l}
\text { if }\left\{x_{n}\right\} \subset X \text { is a increasing sequence } \\
\text { with } x_{n} \rightarrow x \in X \text {, then } x_{n} \prec x \text { for all } n
\end{array}\right.
$$

holds. If there exists $x_{0} \in X$ such that $x_{0} \leqslant F x_{0}$, then there exists $x \in X$ such that $F x=$ $x$. Moreover, $p(x, x)=0$.

Theorem 1.5 (Altun and Erduran [3]) Let $(X, \preccurlyeq)$ be a partially ordered set and suppose that there is a partial metric $p$ on $X$ such that $(X, p)$ is a complete partial metric space. Suppose $F: X \rightarrow X$ is a continuous and non-decreasing mapping such that 


$$
p(F x, F y) \leq \varphi\left(\max \left\{p(x, y), \frac{1}{2}[p(x, F x)+p(y, F y)], \frac{1}{2}[p(x, F y)+p(y, F x)]\right\}\right)
$$

for all $x, y \in X$ with $y \leqslant x$, where $\phi:[0,+\infty) \rightarrow[0,+\infty)$ satisfies conditions (i)-(ii) in Theorem 1.2. If there exists $x_{0} \in X$ such that $x_{0} \leqslant F x_{0}$, then there exists $x \in X$ such that $F x=x$. Moreover, $p(x, x)=0$. If we suppose that for all $x, y \in X$ there exists $z \in$ $X$, which is comparable to $x$ and $y$, we obtain uniqueness of the fixed point of $F$.

Altun et al. [4], Altun and Erduran [3] and many authors have obtained fixed-point theorems for contractions under the assumption that a comparison function $\phi:[0$, $+\infty) \rightarrow[0,+\infty)$ is non-decreasing and such that $\sum_{n=1}^{\infty} \varphi^{n}(t)<\infty$ for each $t>0$ (see, e. g., [13] and the references in $[11,18]$-Added in proof). However, the latter condition is strong and rather hard to verify in practice, though some examples and general criteria for this convergence are known (see, e.g., $[3,44]$ ). So a natural question arises whether this strong condition can be omitted in partial metric fixed-point theory.

The aims of this paper is to establish coincidence and common fixed-point theorems in ordered partial metric spaces with a function $\phi$ satisfying the condition $\phi(t)<t$ for all $t>0$, which is weaker than the condition $\sum_{n=1}^{\infty} \varphi^{n}(t)<\infty$. Presented theorems generalize and extend to a pair of mappings the results of Altun and Erduran [3], Altun et al. [4], Matthews [22] and many other known corresponding theorems.

\section{Main results}

We start this section by some preliminaries.

Definition 2.1 (Altun and Erduran [3]) Let $(X, p)$ be a partial metric space, $F: X \rightarrow$ $X$ be a given mapping. We say that $F$ is continuous at $x_{0} \in X$, if for every $\varepsilon>0$, there exists $\delta>0$ such that $F\left(B_{p}\left(x_{0}, \delta\right)\right) \subseteq B_{p}\left(F x_{0}, \varepsilon\right)$.

The following result is easy to check.

Lemma 2.1 Let $(X, p)$ be a partial metric space, $F: X \rightarrow X$ be a given mapping. Suppose that $F$ is continuous at $x_{0} \in X$. Then, for all sequence $\left\{x_{n}\right\} \subset X$, we have

$$
x_{n} \rightarrow x_{0} \Rightarrow F x_{n} \rightarrow F x_{0} .
$$

Definition 2.2 (Ćirić et al. [11]) Let $(X, \preccurlyeq)$ be a partially ordered set and $F, g: X \rightarrow$ $X$ are mappings of $X$ into itself. One says $F$ is $g$-non-decreasing if for $x, y \in X$, we have

$$
g x \preccurlyeq g y \Rightarrow F x \preccurlyeq F y .
$$

We introduce the following definition.

Definition 2.3 Let $(X, p)$ be a partial metric space and $F, g: X \rightarrow X$ are mappings of $X$ into itself. We say that the pair $\{F, g\}$ is partial compatible if the following conditions hold:

(b1) $p(x, x)=0 \Rightarrow p(g x, g x)=0$,

(b2) $\lim _{n \rightarrow+\infty} p\left(F g x_{n}, g F x_{n}\right)=0$, whenever $\left\{x_{n}\right\}$ is a sequence in $X$ such that $F x_{n} \rightarrow t$ and $g x_{n} \rightarrow t$ for some $t \in X$.

It is clear that Definition 2.3 extends and generalizes the notion of compatibility introduced by Jungck [21].

Define by $\varphi$ the set of functions $\phi:[0,+\infty) \rightarrow[0,+\infty)$ satisfying the following conditions:

(c1) $\phi$ is continuous and non-decreasing,

(c2) $\phi(t)<t$ for each $t>0$. 
Now, we are ready to state and prove our first result.

Theorem 2.1 Let $(X, \preccurlyeq)$ be a partially ordered set and suppose that there is a partial metric $p$ on $X$ such that $(X, p)$ is a complete partial metric space. Let $F, g: X \rightarrow X$ be two continuous self-mappings of $X$ such that $F X \subseteq g X, F$ is a g-non-decreasing mapping, the pair $\{F, g\}$ is partial compatible, and

$$
p(F x, F y) \leq \varphi\left(\max \left\{p(g x, g y), p(g x, F x), p(g y, F y), \frac{1}{2}[p(g x, F y)+p(g y, F x)]\right\}\right)
$$

for all $x, y \in X$ for which $g y \leqslant g x$, where a function $\phi \in \varphi$. If there exists $x_{0} \in X$ with $g x_{0} \leqslant F x_{0}$, then $F$ and $g$ have a coincidence point, that is, there exists $x \in X$ such that $F x=g x$. Moreover, we have $p(x, x)=p(F x, F x)=p(g x, g x)=0$.

Proof. Let $x_{0} \in X$ such that $g x_{0} \leqslant F x_{0}$. Since $F X \subseteq g X$, we can choose $x_{1} \in X$ so that $g x_{1}=F x_{0}$. Again, from $F X \subseteq g X$, there exists $x_{2} \in X$ such that $g x_{2}=F x_{1}$. Continuing this process, we can choose a sequence $\left\{x_{n}\right\} \subset X$ such that

$$
g x_{n+1}=F x_{n}, \quad \forall n \geq 0 .
$$

Since $g x_{0} \leqslant F x_{0}$ and $F x_{0}=g x_{1}$, then $g x_{0} \leqslant g x_{1}$. Since $F$ is a $g$-non-decreasing mapping, we have $F x_{0} \leqslant F x_{1}$, that is, $g x_{1} \leqslant g x_{2}$. Again, using that $F$ is a $g$-non-decreasing mapping, we have $F x_{1} \preccurlyeq F x_{2}$, that is, $g x_{2} \leqslant g x_{3}$. Continuing this process, we get

$$
g x_{1} \preccurlyeq g x_{2} \preccurlyeq g x_{3} \preccurlyeq \cdots \preccurlyeq g x_{n} \preccurlyeq g x_{n+1} \preccurlyeq \cdots
$$

Suppose that there exists $n \in N$ such that $p\left(F x_{n}, F x_{n+1}\right)=0$. This implies that $F x_{n}=$ $F x_{n+1}$, that is, $g x_{n+1}=F x_{n+1}$. Then, $x_{n+1}$ is a coincidence point of $F$ and $g$, and so we have finished the proof. Thus, we can assume that

$$
p\left(F x_{n}, F x_{n+1}\right)>0, \quad \forall n \in \mathbb{N} .
$$

We will show that

$$
p\left(F x_{n}, F x_{n+1}\right) \leq \varphi\left(p\left(F x_{n-1}, F x_{n}\right)\right) \text { for all } n \geq 1 .
$$

Using (2) and applying the considered contraction (1) with $x=x_{n}$ and $y=x_{n+1}$, we get

$$
\begin{aligned}
& p\left(F x_{n}, F x_{n+1}\right) \leq \\
& \varphi\left(\max \left\{p\left(g x_{n}, g x_{n+1}\right), p\left(F x_{n}, g x_{n}\right), p\left(F x_{n+1}, g x_{n+1}\right), \frac{1}{2}\left[p\left(g x_{n}, F x_{n+1}\right)+p\left(F x_{n}, g x_{n+1}\right)\right]\right\}\right) \\
& =\varphi\left(\max \left\{p\left(F x_{n-1}, F x_{n}\right), p\left(F x_{n+1}, F x_{n}\right), \frac{1}{2}\left[p\left(F x_{n-1}, F x_{n+1}\right)+p\left(F x_{n}, F x_{n}\right)\right]\right\}\right) \\
& \leq \varphi\left(\max \left\{p\left(F x_{n-1}, F x_{n}\right), p\left(F x_{n+1}, F x_{n}\right), \frac{1}{2}\left[p\left(F x_{n-1}, F x_{n}\right)+p\left(F x_{n}, F x_{n+1}\right)\right]\right\}\right) .
\end{aligned}
$$

Hence, as

$$
p\left(F x_{n}, F x_{n}\right)+p\left(F x_{n-1}, F x_{n+1}\right) \leq p\left(F x_{n-1}, F x_{n}\right)+p\left(F x_{n}, F x_{n+1}\right)
$$

and $\phi$ is non-decreasing, we have

$$
p\left(F x_{n}, F x_{n+1}\right) \leq \varphi\left(\max \left\{p\left(F x_{n-1}, F x_{n}\right), p\left(F x_{n+1}, F x_{n}\right)\right\}\right) .
$$


If we suppose that $\max \left\{p\left(F x_{n-1}, F x_{n}\right), p\left(F x_{n+1}, F x_{n}\right)\right\}=p\left(F x_{n+1}, F x_{n}\right)$, then from (5),

$$
p\left(F x_{n}, F x_{n+1}\right) \leq \varphi\left(p\left(F x_{n+1}, F x_{n}\right)\right) .
$$

Using (3) and the fact that $\phi(t)<t$ for all $t>0$, we have

$$
p\left(F x_{n}, F x_{n+1}\right) \leq \varphi\left(p\left(F x_{n+1}, F x_{n}\right)\right)<p\left(F x_{n+1}, F x_{n}\right),
$$

a contradiction. Therefore,

$$
\max \left\{p\left(F x_{n-1}, F x_{n}\right), p\left(F x_{n+1}, F x_{n}\right)\right\}=p\left(F x_{n-1}, F x_{n}\right),
$$

and so from (5),

$$
p\left(F x_{n}, F x_{n+1}\right) \leq \varphi\left(p\left(F x_{n-1}, F x_{n}\right)\right) .
$$

Thus, we proved (4).

Since $\phi$ is non-decreasing, repeating the inequality (4) $n$ times, we get

$$
p\left(F x_{n}, F x_{n+1}\right) \leq \varphi^{n}\left(p\left(F x_{0}, F x_{1}\right)\right), \quad \forall n \in \mathbb{N} .
$$

Letting $n \rightarrow+\infty$ in the inequality (6) and using the fact that $\phi^{n}(t) \rightarrow 0$ as $n \rightarrow+\infty$ for all $t>0$, we obtain

$$
\lim _{n \rightarrow+\infty} p\left(F x_{n}, F x_{n+1}\right)=0
$$

On the other hand, we have

$$
\begin{aligned}
& p^{s}\left(F x_{n}, F x_{n+1}\right)=2 p\left(F x_{n}, F x_{n+1}\right)-p\left(F x_{n}, F x_{n}\right)-p\left(F x_{n+1}, F x_{n+1}\right) \\
& \leq 2 p\left(F x_{n}, F x_{n+1}\right) .
\end{aligned}
$$

Letting $n \rightarrow+\infty$ in this inequality, by (7), we get

$$
\lim _{n \rightarrow+\infty} p^{s}\left(F x_{n}, F x_{n+1}\right)=0 .
$$

Now, we shall prove that $\left\{F x_{n}\right\}$ is a Cauchy sequence in the metric space $\left(X, p^{s}\right)$. Suppose, to the contrary, that $\left\{F x_{n}\right\}$ is not a Cauchy sequence in $\left(X, p^{s}\right)$. Then, there exists $\varepsilon>0$ such that for each positive integer $k$, there exist two sequences of positive integers $\{m(k)\}$ and $\{n(k)\}$ such that

$$
n(k)>m(k)>k \text { and } \quad p^{s}\left(F x_{m(k)}, F x_{n(k)}\right) \geq \varepsilon .
$$

Since $p^{s}(x, y) \leq 2 p(x, y)$ for all $x, y \in X$, from (9), for all positive integer $k$, we have

$$
n(k)>m(k)>k \text { and } p\left(F x_{m(k)}, F x_{n(k)}\right) \geq \frac{\varepsilon}{2} \text {. }
$$

Without loss of generality, we can suppose that also

$$
n(k)>m(k)>k, \quad p\left(F x_{m(k)}, F x_{n(k)}\right) \geq \frac{\varepsilon}{2}, \quad p\left(F x_{m(k)}, F x_{n(k)-1}\right)<\frac{\varepsilon}{2} .
$$

From (10) and the triangular inequality (that holds for a partial metric), we have

$$
\begin{aligned}
\frac{\varepsilon}{2} & \leq p\left(F x_{m(k)}, F x_{n(k)}\right) \\
& \leq p\left(F x_{m(k)}, F x_{n(k)-1}\right)+p\left(F x_{n(k)-1}, F x_{n(k)}\right)-p\left(F x_{n(k)-1}, F x_{n(k)-1}\right) \\
& <\frac{\varepsilon}{2}+p\left(F x_{n(k)-1}, F x_{n(k)}\right) .
\end{aligned}
$$


Letting $k \rightarrow+\infty$ and using (7), we get

$$
\lim _{k \rightarrow+\infty} p\left(F x_{m(k)}, F x_{n(k)}\right)=\frac{\varepsilon}{2} .
$$

Again, using the triangular inequality, we obtain

$$
\begin{aligned}
\frac{\varepsilon}{2} & \leq p\left(F x_{m(k)}, F x_{n(k)}\right) \leq p\left(F x_{m(k)}, F x_{m(k)-1}\right)+p\left(F x_{m(k)-1}, F x_{n(k)}\right) \\
& \leq p\left(F x_{m(k)}, F x_{m(k)-1}\right)+p\left(F x_{n(k)}, F x_{m(k)}\right)+p\left(F x_{m(k)-1}, F x_{m(k)}\right) .
\end{aligned}
$$

Letting $k \rightarrow+\infty$ in this inequality, and using (11) and (7), we get

$$
\frac{\varepsilon}{2} \leq \lim _{k \rightarrow+\infty} p\left(F x_{n(k)}, F x_{m(k)-1}\right) \leq \frac{\varepsilon}{2} .
$$

Hence,

$$
\lim _{k \rightarrow+\infty} p\left(F x_{n(k)}, F x_{m(k)-1}\right)=\frac{\varepsilon}{2} .
$$

On the other hand, we have

$$
p\left(F x_{n(k)}, F x_{m(k)}\right) \leq p\left(F x_{n(k)}, F x_{n(k)+1}\right)+p\left(F x_{n(k)+1}, F x_{m(k)}\right) .
$$

From (1) with $x=x_{n}$ and $y=x_{n+1}$, we get

$$
\begin{aligned}
& p\left(F x_{n(k)+1}, F x_{m(k)}\right) \leq \\
& \varphi\left(\operatorname { m a x } \left\{p\left(F x_{n(k)}, F x_{m(k)-1}\right), p\left(F x_{n(k)+1}, F x_{n(k)}\right), p\left(F x_{m(k)}, F x_{m(k)-1}\right),\right.\right. \\
& \left.\left.\frac{1}{2}\left[p\left(F x_{n(k)}, F x_{m(k)}\right)+p\left(F x_{n(k)+1}, F x_{m(k)-1}\right)\right]\right\}\right) \\
& \leq \varphi\left(\operatorname { m a x } \left\{p\left(F x_{n(k)}, F x_{m(k)-1}\right), p\left(F x_{n(k)+1}, F x_{n(k)}\right), p\left(F x_{m(k)}, F x_{m(k)-1}\right),\right.\right. \\
& \left.\left.\frac{1}{2}\left[p\left(F x_{n(k)}, F x_{m(k)}\right)+p\left(F x_{n(k)+1}, F x_{n(k)}\right)+p\left(F x_{n(k)}, F x_{m(k)-1}\right)\right]\right\}\right) \\
& :=\varphi(\xi(k)) .
\end{aligned}
$$

Therefore, from (13) and since $\phi$ is a non-decreasing function, we get

$$
p\left(F x_{n(k)}, F x_{m(k)}\right) \leq p\left(F x_{n(k)}, F_{n(k)+1}\right)+\varphi(\xi(k)) .
$$

Letting $k \rightarrow+\infty$ in the above inequality, using (7), (11), (12) and the continuity of $\phi$, we have

$$
\frac{\varepsilon}{2} \leq \varphi\left(\frac{\varepsilon}{2}\right)<\frac{\varepsilon}{2}
$$

a contradiction. Thus, our supposition that $\left\{F x_{n}\right\}$ is not a Cauchy sequence was wrong. Therefore, $\left\{F x_{n}\right\}$ is a Cauchy sequence in the metric space $\left(X, p^{s}\right)$, and so we have

$$
\lim _{m, n \rightarrow+\infty} p^{s}\left(F x_{n}, F x_{m}\right)=0 .
$$

Now, since $(X, p)$ is complete, then from Lemma $1.1,\left(X, p^{s}\right)$ is a complete metric space. Therefore, the sequence $\left\{F x_{n}\right\}$ converges to some $x \in X$, that is,

$$
\lim _{n \rightarrow+\infty} p^{s}\left(F x_{n}, x\right)=\lim _{n \rightarrow+\infty} p^{s}\left(g x_{n+1}, x\right)=0 .
$$


From the property $(b)$ in Lemma 1.1, we have

$$
p(x, x)=\lim _{n \rightarrow+\infty} p\left(F x_{n}, x\right)=\lim _{n \rightarrow+\infty} p\left(g x_{n+1}, x\right)=\lim _{m, n \rightarrow+\infty} p\left(F x_{n}, F x_{m}\right) .
$$

On the other hand, from property (p2) of a partial metric, we have

$$
p\left(F x_{n}, F x_{n}\right) \leq p\left(F x_{n}, F x_{n+1}\right) \text { for all } n \in \mathbb{N} .
$$

Letting $n \rightarrow+\infty$ in the above inequality and using (7), we obtain

$$
\lim _{n \rightarrow+\infty} p\left(F x_{n}, F x_{n}\right)=0 .
$$

Therefore, from the definition of $p^{s}$ and using (14), we get $\lim _{m, n \rightarrow+\infty} p\left(F x_{n}, F x_{m}\right)=$ 0 . Thus, from (15), we have

$$
p(x, x)=\lim _{n \rightarrow+\infty} p\left(F x_{n}, x\right)=\lim _{m, n \rightarrow+\infty} p\left(F x_{n}, F x_{m}\right)=0 .
$$

Now, since $F$ is continuous, from (16) and using Lemma 2.1, we get

$$
\lim _{n \rightarrow+\infty} p\left(F\left(F x_{n}\right), F x\right)=p(F x, F x) .
$$

Using the triangular inequality, we obtain

$$
p(F x, g x) \leq p\left(F x, F\left(F x_{n}\right)\right)+p\left(F\left(g x_{n+1}\right), g\left(F x_{n+1}\right)\right)+p\left(g\left(F x_{n+1}\right), g x\right) .
$$

Letting $n \rightarrow+\infty$ in the above inequality, using (17), (15), (16), the partial compatibility of $\{F, g\}$, the continuity of $g$ and Lemma 2.1 , we have

$$
p(F x, g x) \leq p(F x, F x)+p(g x, g x)=p(F x, F x) .
$$

Now, suppose that $p(F x, g x)>0$. Then, from (1) with $x=y$, we get

$$
p(F x, F x) \leq \varphi(\max \{p(g x, g x), p(F x, g x)\})=\varphi(p(F x, g x))<p(F x, g x) .
$$

Therefore, from (19), we have

$$
p(F x, g x)<p(F x, g x)
$$

a contradiction. Thus, we have $p(F x, g x)=0$, which implies that $F x=g x$, that is, $x$ is a coincidence point of $F$ and $g$. Moreover, from (16) and since the pair $\{F, g\}$ is partial compatible, we have $p(x, x)=0=p(g x, g x)=p(F x, F x)$. This completes the proof.

An immediate consequence of Theorem 2.1 is the following result.

Theorem 2.2 Let $(X, \preccurlyeq)$ be a partially ordered set and suppose that there is a partial metric $p$ on $X$ such that $(X, p)$ is a complete partial metric space. Suppose $F: X \rightarrow X$ is a continuous and non-decreasing mapping (with respect to $\preccurlyeq$ ) such that

$$
p(F x, F y) \leq \varphi\left(\max \left\{p(x, y), p(x, F x), p(y, F y), \frac{1}{2}[p(x, F y)+p(y, F x)]\right\}\right)
$$

for all $x, y \in X$ with $y \leqslant x$, where $\phi:[0,+\infty) \rightarrow[0,+\infty)$ is continuous non-decreasing and $\phi(t)<t$ for all $t>0$. If there exists $x_{0} \in X$ such that $x_{0} \leqslant F x_{0}$, then there exists $x \in$ $X$ such that $F x=x$. Moreover, $p(x, x)=0$.

Proof. Putting $g x=I x=x$ in Theorem 2.1, we obtain Theorem 2.2.

Now we shall present an example in which $F: X \rightarrow X$ and $\phi:[0,+\infty) \rightarrow[0,+\infty)$ satisfy all hypotheses of our Theorem 2.2, but not the hypotheses of Theorems of 
Altun et al. [4], Altun and Erduran [3] with $\phi$ given in an illustrative example in [3], Matthews [22] and of many other known corresponding theorems.

Before giving our example, we need the following result.

Lemma 2.2 Consider $X=[0,+\infty)$ endowed with the partial metric $p: X \times X \rightarrow[0$, $+\infty)$ defined by $p(x, y)=\max \{x, y\}$ for all $x, y \geq 0$. Let $F: X \rightarrow X$ be a non-decreasing function. If $F$ is continuous with respect to the standard metric $d(x, y)=|x-y|$ for all $x, y \geq 0$, then $F$ is continuous with respect to the partial metric $p$.

Proof. Let $\left\{x_{n}\right\}$ be a sequence in $X$ such that $\lim _{n \rightarrow+\infty} p\left(x_{n}, x\right)=p(x, x)$ for some $x \in$ $X$, that is, $\lim _{n \rightarrow+\infty} \max \left\{x_{n}, x\right\}=x$. Using Lemma 2.1, we have to prove that $\lim _{n \rightarrow+\infty} p$ $\left(F x_{n}, F x\right)=p(F x, F x)$, that is, $\lim _{n \rightarrow+\infty} \max \left\{F x_{n}, F x\right\}=F x$.

Since $F$ is a non-decreasing mapping, we have

$$
\max \left\{F x_{n}, F x\right\}=F\left(\max \left\{x_{n}, x\right\}\right) .
$$

Now, using that $F$ is continuous with respect to the standard metric, we have

$$
\lim _{n \rightarrow+\infty} \max \left\{x_{n}, x\right\}=x \Rightarrow \lim _{n \rightarrow+\infty} F\left(\max \left\{x_{n}, x\right\}\right)=F x .
$$

Therefore, from (21), it follows that

$$
\lim _{n \rightarrow+\infty} \max \left\{F x_{n}, F x\right\}=F x .
$$

This makes end to the proof.

Example 2.1 Let $X=[0,+\infty)$ and $(X, p)$ be a complete partial metric space, where $p$ : $X \times X \rightarrow \mathbb{R}^{+}$is defined by $p(x, y)=\max \{x, y\}$. Let us define a partial order $\leqslant$ on $X$ as follows:

$$
x \preccurlyeq y \Leftrightarrow x=y \text { or }(x, y \in[0,1) \text { with } x \leq y) .
$$

Define $F: X \rightarrow X$ by

$$
F(x)=\left\{\begin{array}{l}
\frac{x}{1+x} \text { if } x \in[0,1), \\
\frac{\sqrt{x}}{2} \quad \text { if } x \geq 1,
\end{array}\right.
$$

and let $\phi:[0,+\infty) \rightarrow[0,+\infty)$ be defined by

$$
\varphi(t)= \begin{cases}\frac{t}{1+t} & \text { if } t \in(0,1], \\ \frac{t}{2} & \text { if } t>1 .\end{cases}
$$

Clearly the function $\phi \in \varphi$, that is, $\phi$ is continuous non-decreasing and $\phi(t)<t$ for each $t>0$. On the other hand, using Lemma 2.2, since $F$ is non-decreasing (with respect to the usual order) and continuous in $X$ with respect to the standard metric, then it is continuous with respect to the partial metric $p$. The function $F$ is also non-decreasing with respect to the partial order $\leqslant$.

We now show that $F$ satisfies the nonlinear contractive condition (20) for all $x, y \in X$ with $y \leqslant x$. By definition of $F$, we have 


$$
\begin{aligned}
p(F x, F y) & =\max \left\{\frac{x}{1+x}, \frac{y}{1+y}\right\} \\
& =\frac{x}{1+x} \\
& =\varphi(\max \{x, y\}) \\
& =\varphi(p(x, y)) .
\end{aligned}
$$

Thus,

$$
p(F x, F y) \leq \varphi\left(\max \left\{p(x, y), p(F x, x), p(F y, y), \frac{1}{2}[p(x, F y)+p(F x, y)]\right\}\right) .
$$

Therefore, the contractive condition (20) is satisfied for all $x, y \in X$ for which $y \leqslant x$. Also, for $x_{0}=0$, we have $x_{0} \leqslant F x_{0}$.

Therefore, all hypotheses of Theorem 2.2 are satisfied and $F$ has a fixed point. Note that it is easy to see that the hypothesis (23) as well as all other hypotheses in Theorems 2.3 and 2.4 below is also satisfied.

Observe that in this example, $\phi$ does not satisfy the condition $\sum_{n=1}^{\infty} \varphi^{n}(t)<\infty$ for each $t>0$ of Theorems in $[3,4]$. Indeed, let $t_{0} \in(0,1]$ be arbitrary. Then, it is easy to show by induction that $\phi^{n}\left(t_{0}\right)=t_{0} /\left(1+n t_{0}\right)$. Thus,

$$
\sum_{n=1}^{\infty} \varphi^{n}\left(t_{0}\right)=\sum_{n=1}^{\infty} \frac{t_{0}}{1+n t_{0}}=+\infty
$$

Note that F does not satisfy the contractive condition (20) in Theorem 2.2 with a function

$$
\varphi(t)=\frac{t^{2}}{1+t} .
$$

This function is given by Altun and Erduran in their illustrative example in [3]. It is easy to show that for $y \leqslant x$,

$$
\begin{aligned}
p(F x, F y) & =\max \left\{\frac{x}{1+x}, \frac{y}{1+y}\right\}=\frac{x}{1+x}>\frac{x^{2}}{1+x} \\
& =\varphi\left(\max \left\{p(x, y), p(x, F x), p(y, F y), \frac{1}{2}[p(x, F y)+p(y, F x)]\right\}\right) \\
& \geq \varphi\left(\max \left\{p(x, y), p(x, F x), p(y, F y), \frac{1}{2}[p(x, F y)+p(y, F x)]\right\}\right) .
\end{aligned}
$$

Now, we will prove the following result.

Theorem 2.3 Let $(X, \preccurlyeq)$ be a partially ordered set and suppose that there is a partial metric $p$ on $X$ such that $(X, p)$ is a complete partial metric space. Let $F, g: X \rightarrow X$ be two self-mappings of $X$ such that $F X \subseteq g X, F$ is a g-non-decreasing mapping and,

$$
p(F x, F y) \leq \varphi\left(\max \left\{p(g x, g y), p(g x, F x), p(g y, F y), \frac{1}{2}[p(g x, F y)+p(g y, F x)]\right\}\right)
$$

for all $x, y \in X$ for which $g x>g y$, where $\phi \in \varphi$. Also suppose

$$
\left\{\begin{array}{l}
\text { if }\left\{g x_{n}\right\} \subset X \text { is a increasing sequence } \\
\text { with } g x_{n} \rightarrow g z \in g X \text {, then } g x_{n} \prec g z, g z \preccurlyeq g(g z) \text { for all } n
\end{array}\right.
$$


holds. Also suppose $g X$ is closed. If there exists $x_{0} \in X$ with $g x_{0} \leqslant F x_{0}$, then $F$ and $g$ have a coincidence point $x \in X$ such that $p(F x, F x)=p(g x, g x)=0$. Further, if $F$ and $g$ commute at their coincidence points, then $F$ and $g$ have a common fixed point.

Proof. Denote

$$
M[F, g](x, y):=\max \left\{p(g x, g y), p(g x, F x), p(g y, F y), \frac{1}{2}[p(g x, F y)+p(g y, F x)]\right\}
$$

for all $x, y \in X$.

As in the proof of Theorem 2.1, we can construct a sequence $\left\{x_{n}\right\}$ in $X$ by $g x_{n+1}=$ $F x_{n}$ for all $n \geq 0$. Also, we can assume that $F x_{n} \neq F x_{n+1}$ for all $n \geq 0$; otherwise, we are finished. Therefore, we have

$$
g x_{1} \prec g x_{2} \prec \cdots \prec g x_{n} \prec g x_{n+1} \prec \cdots
$$

Again, as in the proof of Theorem 2.1, we can show that $\left\{F x_{n}\right\}$ is a Cauchy sequence in the complete metric space $\left(X, p^{s}\right)$, and therefore, there exists $y \in X$ such that

$$
p(y, y)=\lim _{n \rightarrow+\infty} p\left(F x_{n}, y\right)=\lim _{m, n \rightarrow+\infty} p\left(F x_{n}, F x_{m}\right)=0 .
$$

Since $\left\{F x_{n}\right\} \subset g X$ and $g X$ is closed, there exists $x \in X$ such that $y=g x$. From (24) and hypothesis (23), we have

$$
g x_{n} \prec g x \text { for all } n, \quad g x \preccurlyeq g(g x) .
$$

Now, we will show that $x$ is a coincidence point of $F$ and $g$. Using the triangular inequality, we have

$$
p(g x, F x) \leq p\left(g x, g x_{n+1}\right)+p\left(F x_{n}, F x\right) .
$$

From (26), using the considered contraction, we have

$$
p\left(F x, F x_{n}\right) \leq \varphi\left(M[F, g]\left(x, x_{n}\right)\right) .
$$

Thus,

$$
p(g x, F x) \leq p\left(g x, F x_{n}\right)+\varphi\left(M[F, g]\left(x, x_{n}\right)\right) .
$$

Now, we have

$$
\begin{aligned}
& M[F, g]\left(x, x_{n}\right)= \\
& \max \left\{p\left(g x, F x_{n-1}\right), p(F x, g x), p\left(F x_{n}, F x_{n-1}\right), \frac{1}{2}\left[p\left(g x, F x_{n}\right)+p\left(F x, F x_{n-1}\right)\right]\right\} \\
& \leq \max \left\{p\left(g x, F x_{n-1}\right), p(F x, g x), p\left(F x_{n}, F x_{n-1}\right),\right. \\
& \left.\frac{1}{2}\left[p\left(g x, F x_{n}\right)+p(F x, g x)+p\left(g x, F x_{n-1}\right)\right]\right\} .
\end{aligned}
$$

Since $\phi$ is a non-decreasing function, using (25), the above inequality and $n \rightarrow+\infty$ in (27), we get

$$
p(g x, F x) \leq \varphi(p(g x, F x)) .
$$

If $p(g x, F x)>0$, we obtain $p(g x, F x) \leq \phi(p(g x, F x))<p(g x, F x)$ : a contradiction. We deduce that $p(g x, F x)=0$, which implies that $g x=F x$, that is, $x$ is a coincidence point of $F$ and $g$. 
Suppose now that $F$ and $g$ commute at $x$. Set $w=F x=g x$. Then,

$$
F w=F(g x)=g(F x)=g w .
$$

From the hypothesis (23), we have $g x \leqslant g(g x)=g w$. If $g x=g w$, we get $w=g w=F w$, and the proof is finished. Then, suppose that $g x \prec g w$. Applying the considered contraction, we get

$$
p(F w, F x) \leq \varphi(M[F, g](w, x)),
$$

where

$$
\begin{aligned}
& M[F, g](w, x) \\
& =\max \left\{p(g w, g x), p(F w, g w), p(F x, g x), \frac{1}{2}[p(g w, F x)+p(F w, g x)]\right\} \\
& =\max \left\{p(F w, F x), p(F w, F w), p(F x, F x), \frac{1}{2}[p(F w, F x)+p(F w, F x)]\right\} \\
& =\max \{p(F w, F x), p(F w, F w)\} \\
& =p(F w, F x) .
\end{aligned}
$$

Suppose that $p(F w, F x)>0$, From (29), we get

$$
p(F w, F x) \leq \varphi(M[F, g](w, x))=\varphi(p(F w, F x))<p(F w, F x),
$$

which is a contradiction. Thus, we have $p(F w, F x)=0$, which implies that $F w=F x=$ $w$. Therefore, from (28), we have $w=F w=g w$, and $w$ is a common fixed point of $F$ and $g$. This completes the proof.

Remark 2.1 The result given by Theorem 2.3 is also valid if the contraction condition (22) is satisfied for all $x, y \in X$ with $g x \geqslant g y$ and (23) is replaced by

$$
\left\{\begin{array}{l}
\text { if }\left\{g x_{n}\right\} \subset X \text { is a increasing sequence } \\
\text { with } g x_{n} \rightarrow g z \in g X, \quad \text { then } g x_{n} \preccurlyeq g z, g z \preccurlyeq g(g z) \text { for all } n
\end{array}\right.
$$

An immediate consequence of Theorem 2.3 is the following.

Theorem 2.4 Let $(X, \preccurlyeq)$ be a partially ordered set and suppose that there is a partial metric $p$ on $X$ such that $(X, p)$ is a complete partial metric space. Suppose $F: X \rightarrow X$ is a non-decreasing mapping such that

$$
p(F x, F y) \leq \varphi\left(\max \left\{p(x, y), p(x, F x), p(y, F y), \frac{1}{2}[p(x, F y)+p(y, F x)]\right\}\right),
$$

for all $x, y \in X$ with $y<x$, where $\phi:[0,+\infty) \rightarrow[0,+\infty)$ is continuous non-decreasing and $\phi(t)<t$ for all $t>0$. Suppose also that the condition

$$
\left\{\begin{array}{l}
\text { if }\left\{x_{n}\right\} \subset X \text { is a increasing sequence } \\
\text { with } x_{n} \rightarrow x \in X, \text { then } x_{n} \prec x \text { for all } n
\end{array}\right.
$$

holds. If there exists $x_{0} \in X$ such that $x_{0} \leqslant F x_{0}$, then there exists $x \in X$ such that $F x=$ $x$. Moreover, $p(x, x)=0$.

Now, we give a simple example to show that our result given by Theorem 2.3 is more general than Theorem 3.6 of O'Regan and Petrussel [29].

Example 2.2 Let $X=[0,+\infty)$ endowed with the partial metric $p(x, y)=\max \{x, y\}$ for all $x, y \in X$. We endow $X$ with the usual order $\leq$. Consider the mappings $F, g: X \rightarrow X$ 
and $\phi:[0,+\infty) \rightarrow[0,+\infty)$ defined by

$$
F(x)=2 x, \quad g(x)=4 x, \quad \varphi(t)=(3 / 4) t .
$$

Let $y \leq x$. We have

$$
p(F(x), F(y))=F(x)=2 x<3 \cdot \frac{1}{4} \cdot 4 x=\frac{3}{4} p(g(x), g(y))=\varphi(p(g(x), g(y)) .
$$

Then, (22) is satisfied. It is easy to show that all the other hypotheses of Theorem 2.3 are also satisfied. Since $F$ and $g$ commute, we deduce that $F$ and $g$ have a common fixed point $z=0$, that is, $0=F(0)=g(0)$.

On the other hand, if we endow $X$ with the standard metric $d(x, y)=|x-y|$ for all $x$, $y \in X$, we have

$$
d(F(x), F(y))=|F(x)-F(y)|=2|x-y|>\varphi(|x-y|)
$$

for $x \neq y$ and for any $\phi:[0,+\infty) \rightarrow[0,+\infty)$ satisfying $\phi(t)<t$ for $t>0$. Therefore, Theorem 3.6 of O'Regan and Petruşel [29] is not applicable.

Note that $F$ also does not satisfy the contractive conditions in the rest theorems of O’Regan and Petruşel [29].

\title{
Acknowledgements
}

This work was supported by the Ministry of Sciences and technology of Republic Serbia (PROJECT 174025).

\author{
Author details \\ ${ }^{1}$ Université de Tunis, Ecole Supérieure des Sciences et Techniques de Tunis, 5, Avenue Taha Hussein-Tunis, B.P.:56, \\ 1008 Bab Menara, Tunisia ${ }^{2}$ Faculty of Mechanical Engineering, Dositejeva 19, 36000 Kraljevo, Serbia ${ }^{3}$ Faculty of \\ Organizational Science, Jove Ilica 154, 11000 Belgrade, Serbia ${ }^{4} H i g h$ School for Preschool Pedagogues Gnjilane, \\ Karažoržev trg bb, 17520 Bujanovac, Serbia
}

\section{Authors' contributions}

All authors contributed equally and significantly in writing this paper. All authors read and approved the final manuscript.

\section{Competing interests}

The authors declare that they have no competing interests.

Received: 19 January 2011 Accepted: 31 October 2011 Published: 31 October 2011

\section{References}

1. Agarwal, RP, El-Gebeily, MA, O'Regan, D: Generalized contractions in partially ordered metric spaces. Appl Anal. 87(1):109-116 (2008). doi:10.1080/00036810701556151

2. Altun, I, Simsek, H: Some fixed point theorems on ordered metric spaces and application. Fixed Point Theory Appl 2010, 17 (2010). (Article ID 621469)

3. Altun, I, Erduran, A: Fixed point theorems for monotone mappings on partial metric spaces. Fixed Point Theory Appl 2011, 10 (2011). (Article ID 508730). doi:10.1186/1687-1812-2011-10

4. Altun, I, Sola, F, Simsek, H: Generalized contractions on partial metric spaces. Topol Appl. 157(18):2778-2785 (2010). doi:10.1016/j.topol.2010.08.017

5. Amini-Harandi, A, Emami, H: A fixed point theorem for contraction type maps in partially ord ered metric spaces and application to ordinary differential equations. Nonlinear Anal. 72, 2238-2242 (2010). doi:10.1016/j.na.2009.10.023

6. Gnana Bhaskar, T, Lakshmikantham, V: Fixed point theorems in partially ordered metric spaces and applications. Nonlinear Anal. 65, 1379-1393 (2006). doi:10.1016/j.na.2005.10.017

7. Beg, I, Butt, AR: Fixed point for set-valued mappings satisfying an implicit relation in partially ordered metric spaces. Nonlinear Anal. 71, 3699-3704 (2009). doi:10.1016/j.na.2009.02.027

8. Bukatin, M, Kopperman, R, Matthews, S, Pajoohesh, H: Partial metric spaces. Am Math Mon. 116, 708-718 (2009). doi:10.4169/193009709X460831

9. Bukatin, MA, Shorina, SYu: Partial metrics and co-continuous valuations. In: Nivat M, et al (eds.) Foundations of Software Science and Computation Structure, Lecture Notes in Computer Science, vol. 1378, pp. 125-139. Springer, Berlin (1998). doi:10.1007/BFb0053546

10. Cirić, L: Solving the Banach fixed point principle for nonlinear contractions in probabilistic metric spaces. Nonlinear Anal. 72, 2009-2018 (2010). doi:10.1016/j.na.2009.10.001

11. Ćirić, L, Cakić, N, Rajovic, M, Ume, JS: Monotone generalized nonlinear contractions in partially ordered metric spaces Fixed Point Theory Appl 2008, 11 (2008). (Article ID 131294) 
12. Cirić, L, Mihet, D, Saadati, R: Monotone generalized contractions in partially ordered probabilistic metric spaces. Topol Appl. 156(17):2838-2844 (2009). doi:10.1016/j.topol.2009.08.029

13. Fang, J-X: Common fixed point theorems of compatible and weakly compatible maps in Menger spaces. Nonlinear Anal. 71, 1833-1843 (2009). doi:10.1016/j.na.2009.01.018

14. Harjani, J, Sadarangani, K: Fixed point theorems for weakly contractive mappings in partially ordered sets. Nonlinear Anal. 71, 3403-3410 (2009). doi:10.1016/j.na.2009.01.240

15. Harjani, J, Sadarangani, K: Generalized contractions in partially ordered metric spaces and applications to ordinary differential equations. Nonlinear Anal. 72, 1188-1197 (2010). doi:10.1016/j.na.2009.08.003

16. Heckmann, R: Approximation of metric spaces by partial metric spaces. Appl Categ Struct. 7, 71-83 (1999). doi:10.1023/ A:1008684018933

17. Jachymski, J: The contraction principle for mappings on a metric space with a graph. Proc Am Math Soc. 136, 1359-1373 (2008)

18. Jachymski, J: On probabilistic $\varphi$-contractions on Menger spaces. Nonlinear Anal. 73, 2199-2203 (2010). doi:10.1016/j. na.2010.05.046

19. Jachymski, J: Equivalent conditions for generalized contractions on (ordered) metric spaces. Nonlinear Anal. 74(3):768-774 (2011). doi:10.1016/..na.2010.09.025

20. Jleli, M, Samet, B: The Kannan's fixed point theorem in a cone rectangular metric space. J Nonlinear Sci Appl. 2(3):161-167 (2009)

21. Jungck, G: Compatible mappings and common fixed points. Int J Math Math Sci. 9, 771-779 (1986). doi:10.1155/ S0161171286000935

22. Matthews, SG: Partial metric topology. In Proceedings of the 8th Summer Conference on General Topology and Applications, vol. 728, pp. 183-197.New York Academy of Sciences (1994)

23. Matthews, SG: An extensional treatment of lazy data on deadlock. Theor Comput Sci. 151, 195-205 (1995). doi:10.1016/ 0304-3975(95)00051-W

24. Nashine, HK, Samet, B: Fixed point results for mappings satisfying $(\psi, \varphi)$-weakly contractive condition in partially ordered metric spaces. Nonlinear Anal. (2010)

25. Nieto, JJ, Pouso, RL, Rodríuez-López, R: Fixed point theorems in ordered abstract spaces. Proc Am Math Soc. 135, 2505-2517 (2007). doi:10.1090/50002-9939-07-08729-1

26. Nieto, JJ, Rodríguez-López, R: Contractive mapping theorems in partially ordered sets and applications to ordinary differential equations. Order. 22, 223-239 (2005). doi:10.1007/s11083-005-9018-5

27. Nieto, JJ, Rodríguez-López, R: Existence and uniqueness of fixed point in partially ordered sets and applications to ordinary differential equations. Acta Math Sin Engl Ser. 23, 2205-2212 (2007). doi:10.1007/s10114-005-0769-0

28. Oltra, S, Valero, O: Banach's fixed point theorem for partial metric spaces. Rend Istit Mat Univ Trieste. 36, 17-26 (2004)

29. O'Regan, D, Petruşel, A: Fixed point theorems for generalized contractions in ordered metric spaces. J Math Anal Appl. 341, 1241-1252 (2008). doi:10.1016/j.jmaa.2007.11.026

30. Petrusel, A, Rus, IA: Fixed point theorems in ordered L-spaces. Proc Am Math Soc. 134, 411-418 (2006)

31. Rabarison, AF: Partial Metrics. Supervised by ans-Peter A. Künzi, African Institute for Mathematical Sciences (2007)

32. Ran, $A C M$, Reurings, $M C B$ : A fixed point theorem in partially ordered sets and some applications to matrix equations. Proc Am Math Soc. 132(5):1435-1443 (2004). doi:10.1090/50002-9939-03-07220-4

33. Romaguera, S: On Computational Models for the Hyperspace. In Advances in Mathematics Research, vol. 8, pp. 277294.Nova Science Publishers, New York (2009)

34. Romaguera, S: A Kirk type characterization of completeness for partial metric spaces. Fixed Point Theory Appl 2010, 6 (2010). (Article ID 493298)

35. Romaguera, S, Schellekens, M: Duality and quasi-normability for complexity spaces. Appl Gen Topol. 3, 91-112 (2002)

36. Romaguera, S, Valero, O: A quantitative computational model for complete partial metric spaces via formal balls. Math Struct Comput Sci. 19, 541-563 (2009). doi:10.1017/S0960129509007671

37. Rus, IA: Fixed point theory in partial metric spaces. An Univ Vest Timiş Ser Mat-Inform. 2, 149-160 (2008)

38. Rutten, JJMM: Weighted colimits and formal balls in generalized metric spaces. Topol Appl. 89, 179-202 (1998). doi:10.1016/S0166-8641(97)00224-1

39. Saadati, R, Vaezpour, SM, Vetro, P, Rhoades, BE: Fixed point theorems in generalized partially ordered G-metric spaces. Math Comput Model. 52(5-6):797-801 (2010). doi:10.1016/j.mcm.2010.05.009

40. Samet, B: Coupled fixed point theorems for a generalized Meir-Keeler contraction in partially ordered metric spaces Nonlinear Anal. 72, 4508-4517 (2010). doi:10.1016/j.na.2010.02.026

41. Schellekens, MP: A characterization of partial metrizability. Domains are quantifiable. Theor Comput Sci. 305, 409-432 (2003). doi:10.1016/50304-3975(02)00705-3

42. Schellekens, MP: The correspondence between partial metrics and semivaluations. Theor Comput Sci. 315, 135-149 (2004). doi:10.1016/j.tcs.2003.11.016

43. Shakeri, S, ćirić, L, Saadati, R: Common fixed point theorem in partially ordered $\mathcal{L}$-fuzzy metric spaces. Fixed Point Theory Appl 2010, 13 (2010). (Article ID 125082)

44. Thron, WJ: Sequences generated by iteration. Trans Am Math Soc. 96, 38-53 (1960). doi:10.1090/S0002-9947-19600117462-9

45. Valero, O: On Banach fixed point theorems for partial metric spaces. Appl Gen Topol. 6(2):229-240 (2005)

46. Waszkiewicz, P: Quantitative continuous domains. Appl Categ Struct. 11, 41-67 (2003). doi:10.1023/A:1023012924892

47. Waszkiewicz, P: Partial metrisability of continuous posets. Math Struct Comput Sci. 16, 359-372 (2006). doi:10.1017/ S0960129506005196

doi:10.1186/1687-1812-2011-71

Cite this article as: Samet et al:: Common fixed-point results for nonlinear contractions in ordered partial metric spaces. Fixed Point Theory and Applications 2011 2011:71. 